\title{
Impact of a functional-based exercise program on fatigue, quality of life, and muscular endurance in cancer patients: A pilot study
}

\author{
Keauna Braun, Megan Chelberg, Elizabeth Hanke, Samantha Lefaive, Lauren Lenz and Matthew S Wiggins* \\ Department of Kinesiology, University of Wisconsin - Eau Claire, USA
}

\begin{abstract}
Background/Purpose: The purpose of this study is to investigate the impact of a functional-based exercise program on fatigue, quality of life, and muscular endurance in cancer patients.

Methods: Eleven individuals from a Cancer Recovery \& Fitness Program participated in this study. After each individual agreed to participate in the study, baseline measurements were taken for three specific muscular endurance exercises; modified biceps curl, modified sit-to-stand, and a modified chest press. Participants also filled out the Functional Assessment of Cancer Therapy-General (FACT-G) questionnaire on perceived quality of life at baseline testing, and the Fatigue Symptom Inventory (FSI) questionnaire on perceived fatigue every week after one exercise session. After attending the six-week program, the participants were retested using the same methods. Results: Dependent (paired) sample $t$-tests were used to determine if there were significant differences in ME and FACT-G test scores from pretest to post test. Since multiple $t$-tests were being analyzed, the Bonferroni correction was used to control for Type I error, which resulted in an adjusted $p$-value of .0125 . Fatigue was tested using a repeated-measure ANOVA. Significant differences were found in the modified biceps curl $(p=.005)$ and modified chest press tests $(p=.004)$. No significant differences were found for the sit-to-stand test or the FSI and FACT-G questionnaires.
\end{abstract}

Conclusion: A 6-week functional-based exercise program can have a significant impact on muscular endurance with cancer survivors in the area of biceps curls and chest press.

\section{Introduction}

According to Howlader and associates [1], about one in three people (38.5\%) will be diagnosed with some type of cancer within their lifetime in the U.S. Breast cancer is the number one diagnosed cancer in the United States, followed by lung and bronchus cancer, prostate cancer, and colorectal cancer. The occurrence rate of cancer is expected to increase by $45 \%$ between 2010 and 2030, with the greatest increases in older adults and minorities [2]. With cancer rates showing no decline, it is important to continue to investigate treatment and intervention strategies. Common cancer treatments that have been shown to be effective include chemotherapy, radiation therapy, and hormone therapy [3]. Statistics show that after the completion of treatment, $64 \%$ of cancer patients have survived five years or more, $40 \%$ have survived 10 years or more, and $15 \%$ have survived 20 years or more after their initial diagnoses [4]. Upon completion of treatment and the transition into remission, underlying long-term effects frequently remain troublesome for many.

Cancer survivors frequently experience prolonged adverse psychological and physical symptoms including increased risk of anxiety, depression, fatigue, and reduced physical fitness and quality of life (QOL) [5]. QOL is defined as a perceived physical, functional, social, and emotional well-being [6], and generally declines as debilitating diseases such as cancer progress. Additionally, QOL often shares an inverse relationship with cancer-related fatigue (CRF). The National Consortium of Cancer Centers defines CRF as "an unusual persistent subjective sense of tiredness related to cancer or cancer treatment that interferes with usual function" [7]. CRF is an extremely multifaceted and complex experience that has a profound effect on the entirety of a person's physical, emotional, and mental health. Furthermore, CRF interferes with normal daily activities and can have negative social consequences related to one's job and lifestyle [8].

Past studies have revealed that even though there is no definitive exercise prescription for cancer patients, exercise therapy has shown a positive impact on QOL and CRF. LaVoy, Fagundes, and Dantzer found that exercise interventions decreased fatigue levels and improved self-efficacy, while also generating more positive perceptions on QOL ratings [9]. Additional studies have shown improvements in QOL, physical performance, and increases in overall energy from implementing an exercise program for cancer patients undergoing chemotherapy or radiation therapy $[6,10,11]$. Typically, exercise prescriptions have been predominately cardiovascular training such as walking, running, biking, and swimming, and are often paired with resistance training. Programs typically follow a 3-7 days/week of walking or cycling for 35-60 minutes, as well as incorporating some form of resistance training $(60-70 \%$ intensity, 1RM-repetition max,

*Correspondence to: Matthew S Wiggins, Department of Kinesiology, University of Wisconsin- Eau Claire, 105 Garfield Ave., Eau Claire WI, 54701, USA, E-mail: wigginsm@uwec.edu

Key words: cancer patients, muscular endurance, fatigue, quality of life, exercise program

Received: September 04, 2018; Accepted: September 24, 2018; Published: September 28, 2018 
8-12 reps, 2 sets, etc.). This prescription is based on general strength and endurance training [12]. Mustian and colleagues found that implementing a resistance program three times per week using 8-12 repetitions at a moderate intensity resulted in improvements in selfesteem and increases in upper and lower body strength and lean body mass of cancer survivors [3].

As cancer survivorship involves many variables including barriers to exercising, goals and intervention plans should be crafted specifically to the individual [11]. Muscular endurance (ME) is an overlooked parameter of physical testing when investigating exercise protocol options. Since endurance training involves low-to-moderate exercises carried out in a repetitive manner, progressing through an endurancefocused program may allow improvements in endurance across other aspects of life, such as completing activities of daily living (ADLs) without the repercussions of fatigue. In fact, one recent review has shown that performing repetitive physical exercise will help relieve fatigued [9], and should therefore help everyday functioning related to activities of life. Therefore, the purpose of this pilot study is to examine a functional exercise program and its impact on perceived QOL, fatigue, and ME in cancer patients. It is hypothesized that by following an individualized functional exercise program, an increase will be observed in muscular endurance. It is further hypothesized that participants will also experience an increase in their QOL, and a decrease in their fatigue levels.

\section{Methods}

\section{Participants}

Initially, 12 participants agreed to participate in this research study. Of the 12 participants, 92\% $(N=11)$ made it to at least 6 of the 12 exercise sessions. Participant number 07 was dropped from the study because she was not able to complete the post-testing. The average number of sessions missed during our data collection was 3 of the 12 days. For the remaining 11 participants in the study, four were diagnosed with breast cancer, four had multiple myeloma, one individual had Hodgkin's lymphoma, one had thyroid cancer, and one individual was diagnosed with lung cancer. The participants included 8 females and 3 males ranging in age from 42 to 83 (Mean $=68.2$, $S D=11.1$. Time since cancer diagnosis ranged from approximately 3 months to 5 years. Table 1 presents participants' characteristics in more detail. The two requirements for inclusion in the study were that participants be current members of the Cancer Recovery \& Fitness Program at the University of Wisconsin-Eau Claire, and currently have or had some form of cancer. No participants were excluded from the study, and all the subjects were cleared to exercise by their oncologists. All participants signed and agreed to the informed consent documents prior to data collection (Table 1).

\section{Instruments and testing}

In order to assess the patients' CRF, the Fatigue Symptom Inventory (FSI) was distributed at the beginning of each week. The FSI questionnaire was developed by Hann and associates and is a 14item self-report measure designed to evaluate the severity, frequency, and daily patterns of fatigue, as well as perceived interference with QOL [13]. Each item on the FSI is measured on an 11-point scale that assessed the degree to which fatigue, in the previous week, interfered with general level of activity, ability to bathe and dress, normal work activity, ability to concentrate, relations with others, enjoyment of life, and mood. On this scale, zero is "not being fatigued at all," and ten is "being as fatigued as one could be." This assessment provides qualitative
Table 1. Participant characteristics

\begin{tabular}{|l|l|l|l|l|l|}
\hline ID \# & Sex & Age & Cancer Type & Receiving Treatment & Treatment \\
\hline 01 & F & 78 & Right Lung Adenocarcinoma & Yes & 1 \\
\hline 02 & F & 68 & Multiple Myeloma & No & 1,4 \\
\hline 03 & M & 62 & Multiple Myeloma & Yes & 1,4 \\
\hline 04 & M & 71 & Hodgkin's Lymphoma & No & 1 \\
\hline 05 & M & 65 & Multiple Myeloma & Yes & 1 \\
\hline 06 & F & 77 & Thyroid & Yes & 2,3 \\
\hline 08 & F & 58 & Breast & No & 3 \\
\hline 09 & F & 71 & Breast & No & 3 \\
\hline 10 & F & 69 & Breast & No & 3 \\
\hline 11 & F & 41 & Breast & No & 3 \\
\hline 12 & F & 83 & Multiple Myeloma & No & 2 \\
\hline
\end{tabular}

Note: Treatments included are 1-chemotherapy, 2-radiation, 3-surgery, and 4-stem cell transfer.

information about the participant's perceived daily experience with fatigue and cancer.

Psychological well-being was assessed via the Functional Assessment of Cancer Therapy-General Scale (FACT-G) at pre and post-testing stages [14]. The survey assesses the impact of cancer on one's physical, social/family, emotional, and functional QOL domains. It consists of a 27-question evaluation in which the participants answer the questions on a 5-point scale. Zero on the scale signifies "not at all" and four on the scale signifies "very much." A total QOL index can range from 0 to 104 , with higher scores indicating higher perceived QOL. The FACT-G underwent a five-phase validation process showing high coefficients for validity and reliability previously, with 15 cancer specialists providing content validity for the survey [14].

ME was measured using three separate assessments that were individualized for each participant. All participants viewed initial demonstrations of all three exercise tests prior to data collection that included a familiarization process. The two upper body assessments were a biceps curl test and a modified chest press using an appropriate hand weight or resistance band based on the participant's current fitness level. The one lower body test was the sit-to-stand test. Each assessment was tested using a metronome with constant tempo or beats per minute (bpm). The metronome used was derived from a smartphone app called The Metronome by Soundbrenner 2018. The three assessments were carried out in order of: chest press, sit-to-stand, and biceps curl, with each test ending when the subject could no longer keep pace with the metronome bpm cadence. The participants were given an estimated three minutes on average in between tests to fully recover.

\section{Procedures}

The Cancer Recovery \& Fitness Program gained Internal Review Board (IRB) approval for this program to be available to the community from the University of Wisconsin-Eau Claire. All subjects were currently involved in the cancer program which meets twice a week for approximately 40 minutes for each participant. Exercises generally involve using resistance bands and small dumbbells. Each participant starts the exercise session with a short flexibility/range of motion (ROM) warm-up lasting about five minutes. Typically, the exercise portion of the program begins at a low intensity and each participant progresses in intensity and frequency of repetitions from approximately 10 to 15 throughout the training period. Additionally, they also increase from 5 to 6 exercises up to approximately 10 by approximately 2 weeks. Each participant is given an individualized exercise prescription, with most workout sessions including specific 
exercises such as the chest press, row, squat, triceps, leg extensions, biceps, leg abduction and adduction, ROM and two or three balance activities. In addition to the workouts in the Cancer Recovery program, most of the subjects do some form of aerobic activity 2 to 3 days per week on their own. Only the exercise in the Cancer Recovery program is recorded and logged for the participants.

\section{Results}

Data were analyzed to determine differences according to ME (i.e., chest press, bicep curl, sit-to-stand) and QOL assessments using paired sample $t$-tests. Because the statistical analysis consisted of four separate $t$-tests, the Bonferroni correction method was used to control for inflated Type I error by adjusting the $p<.05$ alpha level. Essentially, .05 is divided by the number of tests, resulting in a new alpha level of $p$ $<.0125$. Based on the new level of significance, the paired sample $t$-tests indicated that muscular endurance for chest press $(p=.004)$ and biceps curl $(p=.005)$ significantly increased from pretest to post-test. Sit to stand was not statistically significant $(p=.06)$, but still showed overall improvements in ME from pretest to post-test (Table 2).

No significant results were recorded for overall QOL using the FACT-G questionnaire. Results were recorded individually for the assessment before and after the 6-week muscular endurance program. Responses to the QOL assessment showed a slight 1.19 increase from baseline $(55.36 \pm 7.93)$ to post-test assessment $(56.55 \pm 6.65)$ after the program. The higher scores did indicate a slight overall increase in QOL after the 6-week program (Table 3).

No significant results were found with regard to data from the FSI questionnaire using a repeated-measure $\operatorname{ANOVA}(p>.05)$. As seen in Figure 1, individual scores for all 11 subjects were recorded over the 6-week period for a total of seven measures. While scores did vary each week per participant, there appeared to be a slight decrease in fatigue over the 6-week time interval for several of the subjects (Figure 1).

\section{Discussion}

The purpose of this pilot study was to examine the potential benefits in a functional-based exercise program on muscular endurance (ME), perceived QOL, and fatigue in cancer patients. The initial hypothesis of this study was that by following an individualized exercise program, cancer patients would exhibit increases in ME test repetitions from pretest to posttest. Another anticipated outcome was that fatigue scale ratings would follow a downward trend showing that participants experienced a decrease in their overall fatigue as the program progressed. The final hypothesis was that the functional

Table 2. Overall group results of muscular endurance repetitions for pretest and post-testing

\begin{tabular}{|c|c|c|c|c|}
\hline Fitness Testing & Mean & SD & $t$ & $p$ \\
\hline Chest Press & & & & \\
\hline Pretest & 31.82 & 12.00 & & $0.004^{*}$ \\
\hline Posttest & 49.36 & 21.37 & 3.72 & \\
\hline Sit to Stand & & & & 0.060 \\
\hline Pretest & 34.18 & 21.60 & & \\
\hline Posttest & 49.45 & 29.11 & 2.12 & \\
\hline Bicep Curl & & & & $0.005^{*}$ \\
\hline Pretest & 17.64 & 8.70 & & \\
\hline Posttest & 26.55 & 13.25 & 3.53 & \\
\hline
\end{tabular}

$$
*=p<.0125
$$

Table 3. Overall FACT-G Questionnaire Group Response

\begin{tabular}{|c|c|c|c|c|}
\hline QOL & Mean & SD & $t$ & $p$ \\
\hline Pretest & 55.36 & 7.93 & & \\
\hline Post test & 56.55 & 6.65 & 0.689 & 0.506 \\
\hline
\end{tabular}

\section{Fatigue}

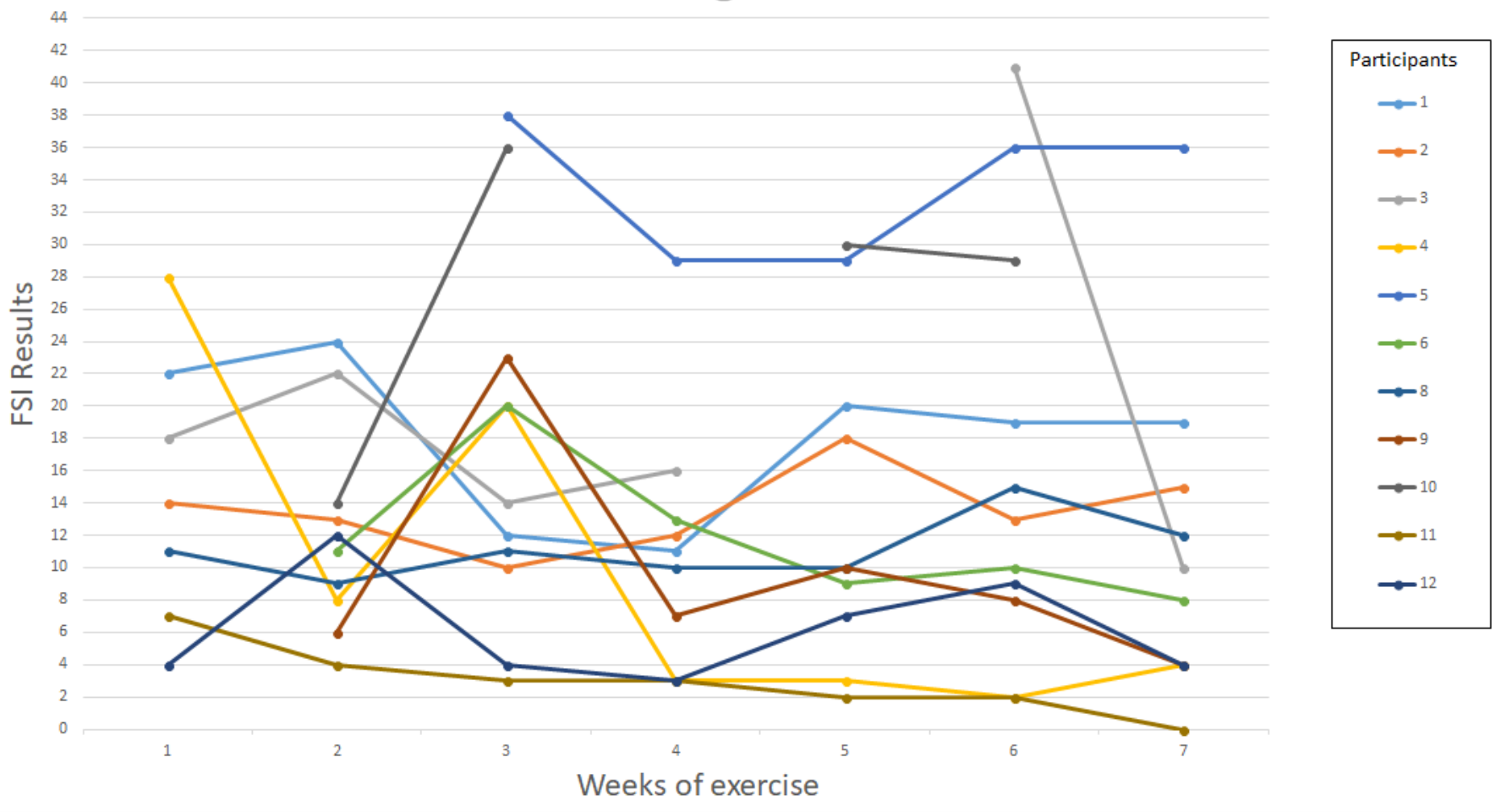

Figure 1. Individual fatigue questionnaire responses by participant 
exercise program would improve overall QOL in the participants that adhered to the exercise program.

Regarding the first hypothesis, two of the three endurance tests showed significant increases from pretest to post-test. Specifically, all 11 participants were able to increase their chest press and biceps curl significantly over the 6-week program. Upper body endurance most likely occurred because the majority of exercises completed in each session were upper body activities. During the workouts, the participants performed 11 general resistance exercises (modified to their fitness and fatigue level) aimed to improve ME and participant-specific characteristics such as shoulder ROM and balance functionality. Of the 11 exercises completed, 7 involved only the upper body. Two of those upper body exercises were the chest press and biceps curl, meaning all participants performed these exercises each week increasing their familiarization of each movement.

Concerning the sit-to-stand test, participants did show improvements in their scores, but failed to reach a significant increase. One reason could be that while 4 of the 11 exercises in the program are lower body focused, most participants did these exercises without incorporating any added resistance or weight. Furthermore, sit-to-stand was not one of the lower body exercises performed by the participants each week, instead being replaced with some type of modified squat exercise. Even though lower body exercises employing no additional weight or resistance still have the potential to increase general ME, it is likely that not enough repetitions or sets were conducted to be able to present significant results in the sit-to-stand test over the 6-week time frame.

Regarding the second hypothesis, the results of the FSI did not provide significant data differences in fatigue levels over the six weeks. As seen in figure 1, the FSI values did not follow any general trend, mostly due to fatigue being a very individualized perception of one's energy levels. Even though this scale has been validated and shown to be reliable, there are other fatigue inventories available that may have been more suitable for this type of special population. In future research that evaluates fatigue levels of cancer patients, an effective alternative to the FSI scale might be The Fatigue Pictogram [15]. This instrument includes only two questions with five figures representing each response option, so in this regard it might be an easier instrument to administer and provide responses. The items assess the intensity of fatigue and the impact of fatigue on daily activities.

Similar to the FSI findings, no significant results were indicated in the values derived from the QOL questionnaire. As with the perceived fatigue ratings, QOL also varied greatly between individuals in this study, and can drastically change over time, especially in cancer patients. While the FACT-G questionnaire has been validated and shown to be reliable in assessing the physical, functional, social and emotional well-being of cancer patients [14], the limited time in this particular research study may have prevented any significant findings to occur. Since the study only lasted 6 total weeks, no trends could be found in the data, and it is possible that significant QOL changes require a longer time-period to improve an individual's psychological well-being. Additionally, the participants missed an average of 3 out of 12 exercise sessions, which may have contributed to the non-significant finding. Therefore, with a longer study intervention, possible intraindividual trends may be identified with this scale.

There were a few study limitations that could have contributed to data inconsistencies. While the number of participants could be considered acceptable for a pilot study, 11 participants is a small sample size. Thus, it is possible that the low number of participants did not provide enough statistical power to find significant values in the sit-to-stand test. More specifically, even though most participants were able to increase their overall repetitions from pretest to post-test, the small sample size could not produce values significant enough to make inferences to a population. Therefore, replicating this study with a larger sample population may produce results with significance in upper and lower-body ME. Another limitation to this study was attendance inconsistencies. Increased adherence to the exercise program may have provided more FSI scale values, potentially allowing for more robust data findings. Finally, the individualized exercise intervention only lasted 6 weeks. Replications of this study should not only include a larger sample size, but also extend the number of weeks in the program to promote even greater increases in ME and QOL.

Overall, this study has demonstrated that an individualized functional-based exercise program for cancer patients is able to increase upper body ME over a short period of time. In fact, one of the most important findings with this research is that ME did increase over a relatively short period of time, as compared with other studies that typically focused on interventions lasting 12 weeks or longer $[10-12,16]$. The increases in overall ME are likely to translate into improvements in completing ADLs with less fatigue. Thus, the current pilot study supports previous research findings that exercise can increase strength and endurance in cancer survivors, and additionally provides evidence that ME gains can be accomplished in as little as six weeks. Hopefully the findings presented in this pilot study will be useful in developing future studies that investigate the effects of an individualized program on physical parameters, measures of functionality, and scales of wellbeing. Since cancer rates are increasing at a progressive rate and patients are continuing to experience CRF and decreases in QOL, it is important that research continues to investigate strategies to ease these symptoms, while also testing and designing exercise programs that increase the physical and psychological benefits related to increases in $\mathrm{ME}$, as well as long-term QOL in cancer survivors.

\section{References}

1. Howlader N, Noone AM, Krapcho M, Miller D, Bishop K, et al. (2014) Cancer stat facts: Cancer of any site. Retrieved February 20, 2018. [http://seer.cancer.gov/statfacts/ $\mathrm{html} / \mathrm{all} . \mathrm{html}]$

2. Smith BD, Smith GL, Hurria A, Hortobagyi GN, Buchholz TA (2009) Future of cancer incidence in the United States: burdens upon an aging, changing nation. J Clin Oncol 27: 2758-2765. [Crossref]

3. Mustian KM, Sprod LK, Palesh OG, Peppone LI, Janelsins MC, et al. (2009) Exercise for the management of side effects and quality of life among cancer survivors. Curr Sports Med Rep 8: 325-330. [Crossref]

4. de Moor JS, Mariotto AB, Parry C, Alfano CM, Padgett L, et al. (2013) Cancer survivors in the United States: Prevalence across the survivorship trajectory and implications for care. Cancer Epidemiol, Biomarkers Prev 22: 561-570. [Crossref]

5. Buffart LM, De Backer IC, Schep G, Vreugdenhil A, Brug J, et al. (2013) Fatigue mediates the relationship between physical fitness and quality of life in cancer survivors. J Sci Med Sport 16: 99-104. [Crossref]

6. Wiggins MS, Simonavice EM (2008) Quality of life benefits: A 12-month exercise \& cancer recovery case study. KAHPERD Journal 44: 16-19.

7. Davis MP, Khoshknabi D, Yue GH (2006) Management of fatigue in cancer patients. Curr Pain Headache Rep 10: 260-269. [Crossref]

8. van Weert E, May AM, Korstjens I, Post WJ, van der Schans CP, et al. (2010) Cancerrelated fatigue and rehabilitation: A randomized controlled multicenter trial comparing physical training combined with cognitive-behavioral therapy with physical training only and with no intervention. Phys Ther 90: 1413-1425. [Crossref]

9. LaVoy ECP, Fagundes CP, Dantzer R (2016) Exercise, inflammation, and fatigue in cancer survivors. Exerc Immunol Rev 22: 82-92. [Crossref]

10. Dimeo F, Schwartz S, Wesel N, Voigt A, Thiel E (2008) Effects of an endurance and resistance exercise program on persistent cancer-related fatigue after treatment. Ann Oncol 19: 1495-1499. [Crossref] 
Braun K (2018) Impact of a functional-based exercise program on fatigue, quality of life, and muscular endurance in cancer patients: A pilot study

11. Wiggins MS, Simonavice EM (2009) Quality of life benefits in cancer survivorship with supervised exercise. Psychol Rep 104: 421-424. [Crossref]

12. McMillan EM, Newhouse IJ (2011) Exercise is an effective treatment modality for reducing cancer-related fatigue and improving physical capacity in cancer patients and survivors: A meta-analysis. Appl Physiol Nutr Metab 36: 892-903. [Crossref]

13. Hann DM, Denniston MM, Baker F (2000) Measurement of fatigue in cancer patients: Further validation of the Fatigue Symptom Inventory. Qual Life Res 9: 847-854. [Crossref]
14. Cella DF, Tulsky DS, Gray G, Sarafian B, Linn E, et al. (1993) The functional assessment of cancer therapy scale: Development and validation of the general measure. J Clin Oncol 11: 570-579. [Crossref]

15. Fitch MI, Bunston T, Bakker D, Mings D, Sevean P (2011) The Fatigue Pictogram: Psychometric evaluation of a new clinical tool. Can Oncol Nurs J 21: 205-210. [Crossref]

16. Wiggins MS (2014) Exercise and perceived quality of life during survivorship: A pilot study comparing traditional and non-traditional cancer recovery programs. Advanced Studies in Medical Sciences 2: 31-36.

Copyright: $@ 2018$ Braun K. This is an open-access article distributed under the terms of the Creative Commons Attribution License, which permits unrestricted use, distribution, and reproduction in any medium, provided the original author and source are credited. 